\title{
Exploratory C star search in the disk of M 31 beyond $30 \mathrm{kpc}^{\star}$
}

\author{
P. Battinelli ${ }^{1}$ and S. Demers ${ }^{2}$ \\ 1 INAF - Osservatorio Astronomico di Roma, Viale del Parco Mellini 84, 00136 Roma, Italia \\ e-mail: battinel@oarhp1.rm.astro.it \\ ${ }^{2}$ Département de Physique, Université de Montréal, CP 6128, Succursale Centre-Ville, Montréal, Québec H3C 3J7, Canada \\ e-mail: demers@astro.umontreal.ca
}

Received 30 June 2004 / Accepted 4 October 2004

\begin{abstract}
In order to investigate how far the intermediate-age population of the disk of M 31 extends we survey $450 \operatorname{arcmin}^{2}$ along its southern major axis, from $35 \mathrm{kpc}$ to $43 \mathrm{kpc}$. We identified a handful of $\mathrm{C}$ stars. We conclude that we have reached the edge of the M 31 disk, at least as defined by intermediate age stars. There is no evidence of density enhancement AGB stars related to the Ferguson clump possibly associated with cluster G1. Radial velocity measures could provide confirmation that the most remote $\mathrm{C}$ star, at $40 \mathrm{kpc}$, is indeed a member of the disk population of M 31 .
\end{abstract}

Key words. galaxies: individual: M 31 - galaxies: local group - galaxies: structure - stars: carbon

\section{Introduction}

In spite of numerous attempts to determine the dynamical mass of nearby galaxies, even for M 31, a galaxy rich in globular clusters, satellites, HII regions and with an extended HI disk, mass estimates are quite far from satisfactory. Indeed we are still discussing if M 31 is or is not the most massive member of the Local Group (Evans \& Wilkinson 2000).

Mass of spiral galaxies are normally determined from their rotation curve obtained from $\mathrm{HI}$ or from $\mathrm{HII}$ regions. In the case of M 31, the HI disk has been observed up to $30 \mathrm{kpc}$ while few HII regions are seen beyond $20 \mathrm{kpc}$ (Kent 1989). Even if both approaches lead to similar curves these methods present different problems: HII offers a good spatial resolution but radial velocities are affected by conspicuous internal kinematics; on the other hand, $\mathrm{HI}$ observations are characterized by a smaller dispersion but a much lower spatial resolution.

In order to extend the survey of the gravitational potential well of M 31, the radial velocity of planetary nebulae (PNe), globular clusters and satellites have been used. PNe and globular clusters are of little help when one wants to reach large distances. Indeed (Battistini et al. 1993), only a handful of globular clusters are farther than $80^{\prime}(20 \mathrm{kpc})$. Only two known globular clusters of M 31 are at more than $30 \mathrm{kpc}$ from its center (Racine 1991). No PNe are known outside this limit.

The Keck telescope has recently been used to obtain radial velocities of M 31's satellites (Evans et al. 2000;

* Based on observations obtained with the Italian Telescopio Nazionale Galileo (TNG) operated on the island of La Palma by the Centro Nazionale Galileo Galilei of the INAF at the Spanish Observatorio del Roque de los Muchachos of the Instituto de Astrofisica de Canarias.
Côté et al. 2000) in order to constraint the mass of M 31 . The mass estimate is accompanied by considerable uncertainty caused by the small size of the data set. This method also relies on the assumption that the selected "satellites" are indeed bound to M 31. This is far from certain for some of the galaxies around M 31. For instance IC 1613 is accepted by the above authors in spite of its projected distance from M 31 of $500 \mathrm{kpc}$.

We have recently (Battinelli et al. 2003) discovered nearly 1000 carbon stars in the outer disk of M 31, up to the edge of its known HI disk (Cram et al. 1980). We surveyed part of the south west major axis corresponding to distances between 17 and $30 \mathrm{kpc}\left(76^{\prime}\right.$ to $\left.130^{\prime}\right)$ from the center. Carbon stars offer the ideal kinematical probes being brighter than the tip of the red giant stars. In M 31 this would correspond to $I \approx 20.4$. With these considerations in minds we present a first step toward a full survey of the periphery of M 31's disk.

The region surveyed in the present paper is well beyond the area of M 31 observed by Walterbos \& Kennicutt (1988), it is in fact close to G1, the brightest M 31 globular cluster, which has been the target of several recent observations. Its surrounding field has been observed by Rich et al. (1996) and more recently by Rich et al. (2004). The position of G1 is shown in Fig. 1, the size of the circle corresponds to its tidal radius (Maylan et al. 2001). The triangle, north of it, indicates the location of its surrounding field. These studies conclude that the field population has a metallicity of $[\mathrm{Fe} / \mathrm{H}]=-0.7$ and does contain intermediate age stars. Furthermore, Reitzel et al. (2004) showed that the radial velocities of red giant stars in the proximity of G1 match the velocity of the HI disk, this implies the presence of a non negligible amount of disk population up to this distance. 


\section{Observations}

The observations, carried on in service mode at the Telescopio Nazionale Galileo, were acquired during a few nights in July and August 2003. They consist in images obtained through $R, I$, $\mathrm{CN}$ and $\mathrm{TiO}$ images. The narrow-band $(F W H M=30 \mathrm{~nm})$ $\mathrm{CN}$ and TiO filters are centered respectively on $808.6 \mathrm{~nm}$ and $768.9 \mathrm{~nm}$. The camera DOLORES is equipped with a Loral thinned and back-illuminated $2048 \times 2048$ CCD with a scale of $0.275 \mathrm{arcsec} / \mathrm{pix}$ corresponding to a field of view of about $9.4 \times$ 9.4.

Equatorial coordinates (J2000.0) of the five target fields are listed in Table 1, along with their angular and absolute distances from the center of M 31. These fields, which overlap slightly are located along the southern major axis of $\mathrm{M}$ 31. Figure 1 sketches their positions relative to the CFH12K fields SW1, and SW2 observed by Battinelli et al. (2003).

The observing $\log$ is presented in Table 2. The seeing ranges from 0.7 to 1.2 arcsec. Exposure times are selected to obtain a $S / N=20$, at $I \approx 22$, a magnitude corresponding to $\approx 2$ mag fainter than the mean magnitude of $\mathrm{C}$ stars in M 31 .

\subsection{Data analysis}

Data reduction was done using DAOPHOT/ALLSTAR on combined images in each filter. The ALLSTAR task of DAOPHOT provides, for each "star" detected, the $\mathrm{Chi}^{2}$ of the psf as well as the sharpness of the image. The sharpness serves to eliminate cosmic rays, blends and background galaxies. Since we target low density fields we expect to observe a non-negligible number of background galaxies. According to Smail et al. (1995) deep galaxy counts we should expect $\sim 150$ galaxies with $I$ magnitude between 20 and 21 in one DOLORES field.

To remove the non-stellar objects we plot, for each image, the $\mathrm{Chi}^{2}$ and the sharpness parameters as a function of the instrumental magnitudes. Objects deviating from the bulk of stars were deleted. This exercise removes between $23 \%$ (in SW3) and $49 \%$ (in SW7) of objects with photometric errors less than 0.100 mag. Objects detected by DAOPHOT at the faint magnitude end have large errors and most of them have quite large sharpness.

Calibration equations were provided by the TNG staff in the following form:

$(R-I)=0.9089(r-i)+0.534-k_{R I} X$,

$I=0.899+i+0.1858(R-I)-k_{I} X$.

We assume standard La Palma extinction coefficients and did not determine them for each night. The zero-point of the narrow band observations was obtained setting the average $(\mathrm{CN}-\mathrm{TiO})$ of blue stars equal to zero following a procedure similar to what adopted by Brewer et al. (1995). This procedure is explained by Letarte et al. (2002) and in the present paper we follow their definition of blue stars as stars in the colour range $0.0<(R-I)_{0}<0.45$. To convert observed $(R-I)$ colours into intrinsic colours we adopt $E(B-V)=0.10$ corresponding to the value for the SW2 field of Battinelli et al. (2003). This
Table 1. South West major axis fields.

\begin{tabular}{cccc}
\hline \hline Field & RA J2000 Dec & $D^{\prime}$ & $D(\mathrm{kpc})$ \\
\hline SW3 & $00: 35: 13+39: 23: 50$ & $141^{\prime}$ & 31.2 \\
SW4 & $00: 34: 47+39: 15: 50$ & $151^{\prime}$ & 33.5 \\
SW5 & $00: 34: 21+39: 07: 50$ & $160^{\prime}$ & 35.5 \\
SW6 & $00: 33: 50+38: 59: 50$ & $170^{\prime}$ & 37.7 \\
SW7 & $00: 33: 19+38: 51: 50$ & $180^{\prime}$ & 39.9 \\
\hline
\end{tabular}

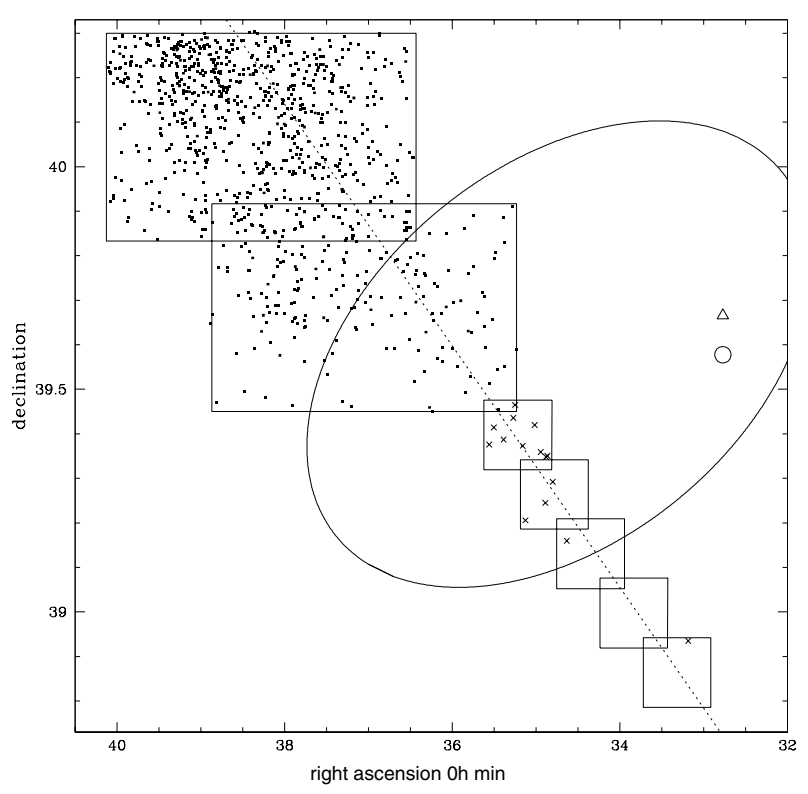

Fig. 1. Schematic representation of the five TNG fields relative to the CFH12K fields. Dots are the known C stars; crosses are the newly discovered $\mathrm{C}$ stars; the dashed line corresponds to the major axis of the disk of M 31. The cluster $\mathrm{G} 1$ is represented by a circle, the triangle is the region called G1 field and the ellipse outlines the Ferguson et al. (2002) clump (see text for details).

corresponds to $E(R-I)=0.073$ and $A_{I}=0.196$ according to Cardarelli et al. (1989). This reddening is not too different from the one adopted by Rich et al. (2004) for the photometry near the cluster G1. As a further check of our calibration we compare the magnitudes and colours of 22 stars, with photometric errors smaller than $0.05 \mathrm{mag}$, which are in the overlap between SW2 and SW3. We found a mean $\Delta(I)=0.06 \pm 0.04$ and for the colour $\Delta(R-I)=0.05 \pm 0.05$. Since the narrow-band filters used at the TNG are wider than those at the CFHT the two $(\mathrm{CN}-\mathrm{TiO})$ indices are not straightforwardly comparable.

\section{Results}

\subsection{The colour-magnitude diagrams}

Figure 2 presents the colour-magnitude diagrams (CMD) of the five fields. Only stars with colour errors less than 0.10 mag are included. The bright end of M 31's RGB is obvious in SW3 and SW4 but less so in the three other fields. The SW7 reaches slightly fainter magnitudes because we have longer exposures for that field. The colour position of the vertical ridge is 
Table 2. Summary of the observations.

\begin{tabular}{|c|c|c|c|c|}
\hline Field & $I$ & $R$ & $\mathrm{CN}$ & $\mathrm{TiO}$ \\
\hline \multicolumn{5}{|l|}{ SW3 } \\
\hline Exposure & $450 \mathrm{~s}$ & $600 \mathrm{~s}$ & $2 \times 750 \mathrm{~s}$ & $2 \times 750 s$ \\
\hline Airmass & 1.28 & 1.25 & 1.18 & 1.51 \\
\hline \multicolumn{5}{|l|}{ SW4 } \\
\hline Exposure & $450 \mathrm{~s}$ & $600 \mathrm{~s}$ & $2 \times 750 \mathrm{~s}$ & $2 \times 750 s$ \\
\hline Airmass & 1.18 & 1.15 & 1.11 & 1.05 \\
\hline \multicolumn{5}{|l|}{ SW5 } \\
\hline Exposure & $450 \mathrm{~s}$ & $600 \mathrm{~s}$ & $2 \times 750 \mathrm{~s}$ & $2 \times 750 s$ \\
\hline Airmass & 1.02 & 1.02 & 1.02 & 1.02 \\
\hline \multicolumn{5}{|l|}{ SW6 } \\
\hline Exposure & $240 s+450 s$ & $300 s+600 s$ & $2 \times 750 \mathrm{~s}$ & $2 \times 750 \mathrm{~s}$ \\
\hline Airmass & 1.15 & 1.14 & 1.03 & 1.09 \\
\hline \multicolumn{5}{|l|}{ SW7 } \\
\hline Exposure & $4 \times 240 s+450 s$ & $2 \times 300 s+600 s$ & $4 \times 750 \mathrm{~s}$ & $2 \times 750 s$ \\
\hline Airmass & 1.08 & 1.09 & 1.03 & 1.06 \\
\hline
\end{tabular}

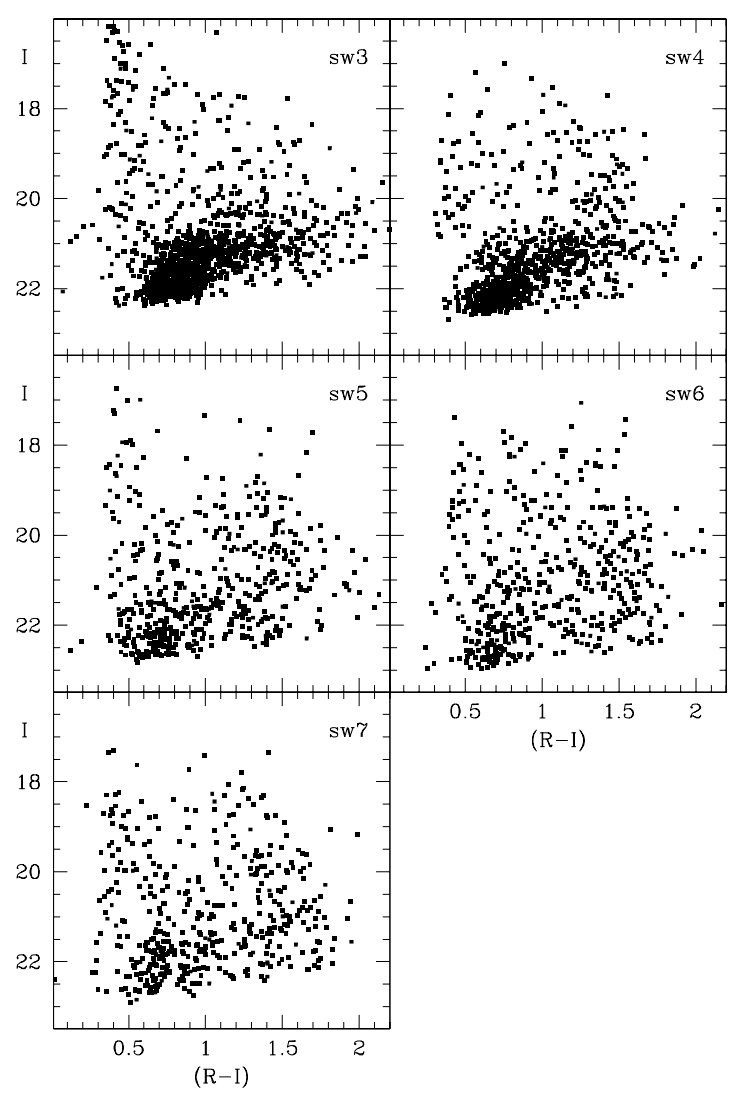

Fig. 2. The colour-magnitude diagrams of the five fields under investigation. Only stars with colour errors $<0.100$ are plotted.

indicative of the reddening in that direction and it appears to be fully consistent with our adopted colour excess. Furthermore,

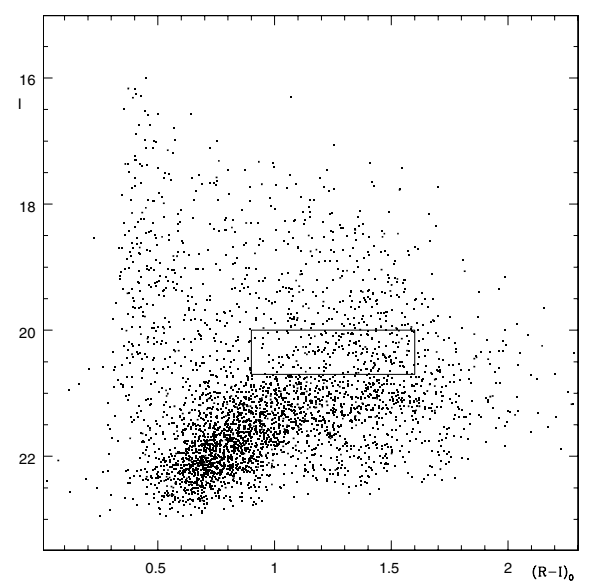

Fig. 3. The AGB star region is outlined on the CMD of the concatenated five fields.

the ridge colour is constant in the five fields suggesting no foreground reddening variation.

Before proceeding with the identification of the $\mathrm{C}$ stars we would like to investigate the extend of the AGB population along the major axis of M 31. To do so, we define a box in the CMD where AGB stars are found. Figure 3 displays the CMD which corresponds to the sum of the five CMD's of Fig. 2. A box, following Ferguson et al. (2002) outlines the region where AGB stars are found. The limits of this box are: $0.9<(R-I)_{0}<$ 1.6 and $20.0<I<20.7$.

Figure 4 presents the number of AGB stars in the five TNG fields as a function of the angular distance along M 31 major axis. The first two points correspond to counts in regions of the size of DOLORES in SW2 (Battinelli et al. 2003). 




Fig. 4. The number of AGB stars in the $88 \operatorname{arcmin}^{2}$ region along the major axis of M 31. The dashed line represents Durrell's et al. (2001) counts in R1.

The dashed line represents the counts in Durrell et al. (2001) region R1 located 7.5 degrees from M 31. We assume that these counts represent the foreground contribution. We then conclude that there is no appreciable AGB population above the foreground at radial distances exceeding $\sim 150^{\prime}$, thus SW5, SW6 and SW7 should contain few if any C stars. It is interesting to note that these fields are partly or completely out of Ferguson's density enhancement. We remind the reader however that the observed density enhancement near G1, outlined in our Fig. 1, is for RGB stars while AGB stars show a marginal enhancement. In any case, Fig. 4 demonstrates that we seem to have reached the edge of the intermediate-age disk and that very few if any $\mathrm{C}$ stars are expected beyond the surveyed area. In Sect. 4. we use recent M 33 results to re-evaluate the radial limit of $\mathrm{C}$ stars.

\subsection{The colour-colour diagrams}

The identification of $\mathrm{C}$ stars among the red stars is accomplished thanks to the $(\mathrm{CN}-\mathrm{TiO})$ index, plotted as a function of $(R-I)$ on a colour-colour diagram. Numerous faint stars with poorer photometry tend, however, to pollute the colour-colour diagram that sometimes may lead to spurious identifications of C stars. Therefore, our survey approach, has been to limit the star selection to stars with photometric colour errors (as defined by DAOPHOT) of $\sigma_{\text {colours }}=\left(\sigma_{(R-I)}^{2}+\sigma_{(\mathrm{CN}-\mathrm{TiO})}^{2}\right)^{1 / 2}<0.125$.

Figure 5 presents the five colour-colour diagrams. The two boxes define, following Albert et al. (2000) the regions of $\mathrm{C}$ stars (upper of one) and $\mathrm{M}$ stars (lower one). The diagram of SW3 shows a pronounced scatter within the M box as well as in the $\mathrm{C}$ box. Contrary to the other fields, the $\mathrm{TiO}$ images of SW3 were obtained not on the same night but some 60 days after the $\mathrm{CN}$ observations. We believe that the scatter seen in the red part of the diagram can be explained by the presence of a number of Mira or Semi-regular variables. The exact fraction of red giants and of AGB stars that are variables is not known but from repeated radial velocity measures, Kunkel et al. (1997) concluded that 10 to 20 percent of $\mathrm{C}$ stars are variables.



Fig. 5. The colour-colour diagrams of the five fields. Only stars with $\sigma_{\text {colours }}<0.125$ are plotted (see text). The two boxes define the samples of $\mathrm{C}$ and $\mathrm{M}$ stars.

A total of $15 \mathrm{C}$ stars are found in the surveyed area, their accurate positions along with their photometric properties are listed in Table 3 . We note that in SW3 three C stars show very large $(\mathrm{CN}-\mathrm{TiO})$ colours which are not observed in the other fields. Even though we are dealing with a poor statistics we believe that such colours may be again explained by the mentioned delay between the $\mathrm{CN}$ and the TiO observations: a luminosity fading of a variable stars between the two epochs of observations would result into such large colours. On the other hand, a luminosity increase between the observations might produced artificially small $(\mathrm{CN}-\mathrm{TiO})$ colours for variable stars thus preventing their identification as genuine $\mathrm{C}$ stars. Variable star surveys of Local Group galaxies, currently in progress, such as Baldacci et al. (2004), will provide, in the near future, robust statistics on the $\mathrm{C}$ star variability.

\section{Discussion}

The average $I$ magnitude of the $15 \mathrm{C}$ stars, listed in Table 3, is $\left\langle I_{0}\right\rangle=20.54$, noticeably fainter than $\left\langle I_{0}\right\rangle=19.94 \mathrm{de}-$ termined by Battinelli et al. (2003) for $945 \mathrm{C}$ stars. This fainter $I_{0}$-magnitude matches, as shown in Fig. 6, the observed M 31 C star luminosity trend seen as a function of galactocentric distances. Indeed, ten of our fifteen $\mathrm{C}$ stars fall below the linear regression defined by the Battinelli et al. (2003) sample. A similar trend is seen when the bolometric magnitudes are used. Bolometric corrections are obtained using the 
Table 3. $\mathrm{C}$ stars in the outer disk of $\mathrm{M} 31^{a}$.

\begin{tabular}{ccccccccc}
\hline \hline id & RA & Dec & $I$ & $\sigma_{I}$ & $R-I$ & $\sigma_{R-I}$ & $\mathrm{CN}-\mathrm{TiO}$ & $\sigma_{\mathrm{CN}-\mathrm{TiO}}$ \\
\hline 1 & $0: 33: 11.05$ & $+38: 56: 04.9$ & 20.370 & 0.010 & 1.174 & 0.016 & 0.470 & 0.022 \\
2 & $0: 34: 38.03$ & $+39: 09: 34.6$ & 21.038 & 0.024 & 1.228 & 0.037 & 0.335 & 0.036 \\
3 & $0: 34: 48.01$ & $+39: 17: 32.4$ & 20.716 & 0.021 & 0.991 & 0.028 & 0.331 & 0.029 \\
4 & $0: 34: 51.98$ & $+39: 21: 02.6$ & 20.552 & 0.019 & 1.036 & 0.029 & 0.377 & 0.040 \\
5 & $0: 34: 52.93$ & $+39: 20: 53.5$ & 20.263 & 0.016 & 1.094 & 0.023 & 0.276 & 0.031 \\
6 & $0: 34: 53.37$ & $+39: 14: 41.2$ & 21.096 & 0.027 & 1.257 & 0.048 & 0.316 & 0.045 \\
7 & $0: 34: 56.68$ & $+39: 21: 33.9$ & 21.382 & 0.030 & 1.886 & 0.070 & 0.420 & 0.041 \\
8 & $0: 35: 01.11$ & $+39: 25: 10.8$ & 20.362 & 0.014 & 1.130 & 0.024 & $0.812 \dagger$ & 0.023 \\
9 & $0: 35: 07.62$ & $+39: 12: 20.1$ & 20.749 & 0.019 & 1.066 & 0.028 & 0.391 & 0.030 \\
10 & $0: 35: 09.54$ & $+39: 22: 23.0$ & 20.660 & 0.019 & 1.316 & 0.031 & $0.773 \dagger$ & 0.025 \\
11 & $0: 35: 14.95$ & $+39: 27: 53.5$ & 20.677 & 0.020 & 1.671 & 0.043 & 0.444 & 0.028 \\
12 & $0: 35: 16.33$ & $+39: 26: 07.8$ & 19.854 & 0.011 & 1.224 & 0.017 & 0.269 & 0.022 \\
13 & $0: 35: 23.31$ & $+39: 23: 14.4$ & 20.352 & 0.014 & 1.145 & 0.022 & $0.918 \dagger$ & 0.021 \\
14 & $0: 35: 30.28$ & $+39: 24: 50.9$ & 20.552 & 0.019 & 1.036 & 0.029 & 0.377 & 0.040 \\
15 & $0: 35: 33.60$ & $+39: 22: 32.6$ & 21.044 & 0.024 & 1.934 & 0.064 & 0.629 & 0.025 \\
\hline
\end{tabular}

${ }^{a}$ Units of right ascensions are hours, minutes and seconds, and units of declination are degrees, arcminutes and arcseconds. $\dagger$ : stars in SW3 which $(\mathrm{CN}-\mathrm{TiO})$ are certainly affected by the time lag between observations (see Sect. 3.2).

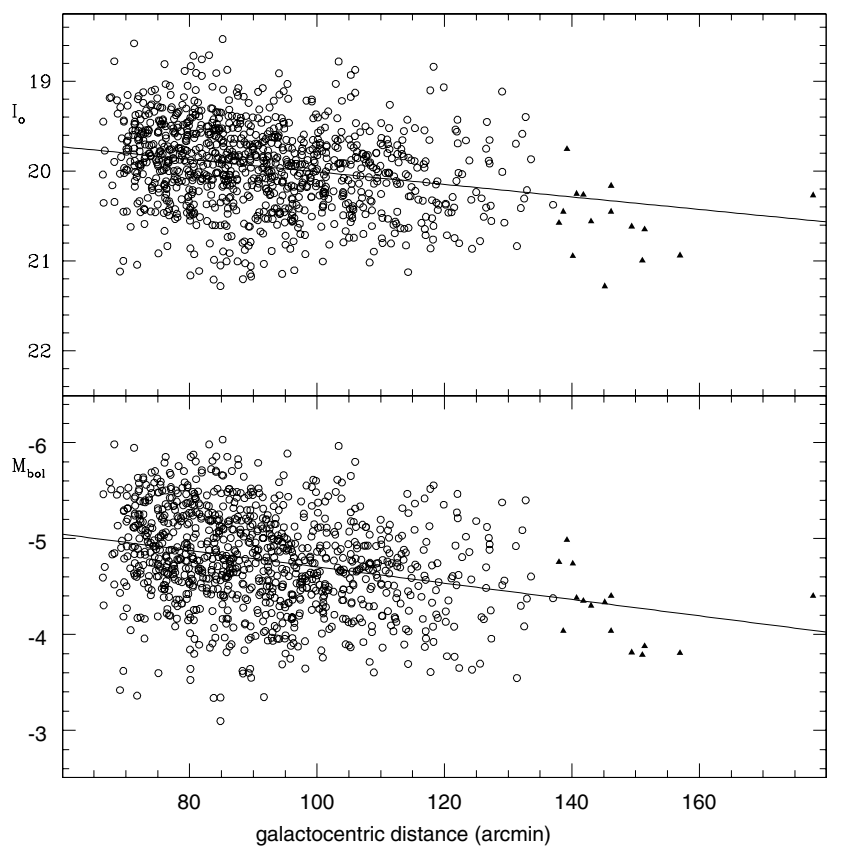

Fig. 6. Luminosities of C stars from Battinelli et al. (2003) (open circles) and from the present paper (filled triangles) as a function of the angular distance from the M 31 center. The straight line is the least square fit to the Battinelli et al. C stars. The upper panel presents the reddening corrected $I$ magnitudes while the lower panel shows the bolometric magnitudes.

Battinelli \& Demers (2005) relation in terms of $(R-I)$ colors based in Table 6 of Bessell et al. (1998). Furthermore, a recent M 31 outer disk survey, using Sloan $r^{\prime}, g^{\prime}$ and $i^{\prime}$ filters, also suggests that bright $\mathrm{C}$ stars are lacking in the outskirt of the disk (Demers \& Battinelli, in preparation). Such magnitude trend is also seen in NGC 6822 (Letarte et al. 2002), albeit less pronounced because of the much shorter distance range. The bright limit of the apparent magnitudes of the LMC C stars, compiled by Kontizas et al. (2001) shows, likewise, an obvious radial trend, over some 8 degrees. Very bright $\mathrm{C}$ stars, confirmed spectroscopically, have been observed in the Large Magellanic Cloud (Richer et al. 1978, 1979; Richer 1981), these stars are in general somewhat bluer than our adopted colour limit. These authors attribute their origin to massive stars. It is quite possible that such massive and younger $\mathrm{C}$ stars are present in the current star forming regions of $\mathrm{M} 31$ while absent from the outskirts where no new stars are formed. On the other hand, one must keep in mind that blending could be responsible for the appearance of brighter $\mathrm{C}$ stars in central regions where the stellar surface density is substantially larger than in the periphery.

The fainter $\mathrm{C}$ stars seen in our fields appears to be quite normal stars. To double check the nature of the fainter $\mathrm{C}$ star candidates, we visually inspected each star on each image. Even star number 7, the faintest of them all, is found in the SW3 images to be perfectly normal and not affected by bright neighbours, cosmic rays nor CCD defects. Faint high latitude carbon stars (FHLCs) have recently been identified, from the Sloan Digital Sky Survey, by Downes et al. (2004) to an apparent magnitude corresponding to $I \sim 20$. Such stars are, however, extremely rare. Indeed, one thousand DOLORES fields would contain only two of them! Moreover, nearly all of them have a $(g-r)<2.0$ thus too blue to be included in our sample with a lower colour limit at $(R-I)_{0}=0.90$.

The small population of outer disk C stars, is reminiscent of the small sample of $\mathrm{C}$ stars recently identified by Harbeck et al. (2004) in a few dwarf spheroidals, satellites of M 31. These stars are, on average fainter than $\mathrm{C}$ stars found by us in dwarf irregular galaxies. Similarly, Kerschbaum et al. (2004) 
also found fainter C stars in And II. The brighter, younger, more massive $\mathrm{C}$ stars must be missing in these small population where star formation has stopped several gigayears ago.

The positive identification of $\mathrm{C}$ stars in this very outer region of the M 31 disk is however of great interest. These stars trace the presence of an intermediate age population even when the signal from stellar counts is lower than the foreground statistical fluctuation (see Fig. 4). Moreover, the discovery of such bright AGB stars offer the opportunity to use them as kinematical probes thus calling for a spectroscopic follow-up aimed to trace the rotation curve on M 31 well beyond the $\mathrm{H}$ I disk.

How far can we expect to see C stars in M 31? To answer this question we look at the observed radial $\mathrm{C}$ star distribution of M 33 (Rowe et al. 2004). In M 33, the surface density profile decreases continuously to $50^{\prime}$ then becomes more or less constant until the number of $\mathrm{C}$ stars becomes too small for meaningful statistics. $50^{\prime}$ correspond to 8.6 times the scale length of the density profile. According to Battinelli et al. (2003), the M $31 \mathrm{C}$ star scale length is $4.9 \mathrm{kpc}$ or $21.9^{\prime}$ implying that, if both $\mathrm{C}$ star populations are distributed in the same fashion, we should see C stars to at least to $188^{\prime}$. Rowe et al. (2004) note, however, that the $\mathrm{C}$ star surface distribution in the outer parts of the disk of M 33 shows irregularities and follows the stellar density of spiral arms. Thus a large scale survey is needed to compensate for the local irregularities which are expected in the periphery of the disk.

Acknowledgements. We thanks the TNG staff for a useful support and for providing us with the colour calibration equations. This research is funded in parts (S.D.) by the Natural Science and Engineering Council of Canada.

\section{References}

Albert, L., Demers, S., \& Kunkel, W. E. 2000, AJ, 119, 2780

Baldacci, L., Rizzi, L., Clementini, G., \& Held, E. V. 2004, A\&A, in press
Battinelli, P., Demers, S., \& Letarte, B. 2003, AJ, 125, 1298

Battinelli, P., \& Demers, S. 2005, A\&A, submitted

Battistini, P. L., Bonoli, F. Casavecchia, M., et al. 1993, A\&A, 272, 77

Bessell, M. S., Castelli, F., \& Plez, B. 1998, A\&A, 333, 231

Brewer, J. P., Richer, H. B., \& Crabtree, D. R. 1995, AJ, 109, 2480

Cardarelli, J. A., Clayton, G. C., \& Mathis, J. S. 1989, ApJ, 345, 245

Côté, P., Mateo, M., Sargent, W. L. W., \& Olszewski, E. W. 2000, ApJ, 537, 91

Cram, T. R., Roberts, M. S., \& Whitehurst, R. N. 1980, A\&AS, 40, 215

Downes, R. A., Margon, B., Anderson, S. F., et al. 2004, AJ, 127, 2838

Durrell, P. R., Harris, W. E., \& Pritchet, C. J. 2001, AJ, 121, 2557

Evans, N. W., \& Wilkinson, M. I. 2000, MNRAS, 316, 929

Evans, N. W., Wilkinson, M. I., Guhathakurta, P., Grebel, E. K., \& Vogt, S. S. 2000, ApJ, 540, 9

Ferguson, A. M. N., Irwin, M. J., Ibata, R. A., Lewis, G. F., \& Tanvir, N. R. 2002, AJ, 124, 1452

Harbeck, D., Gallagher, J. S., \& Grebel, E. K. 2004, AJ, 127, 2711

Kent, S. M. 1989, PASP, 101, 489

Kerschbaum, F., Nowotny, W., Olofsson, H., \& Schwarz, H. E. 2004, A\&A, in press

Kontizas, E., Dapergolas, A., Morgan, D. H., \& Kontizas, M. 2001, A\&A, 369, 932

Kunkel, W. E., Demers, S., Irwin, M. J., \& Albert, L. 1997, ApJ, 488, L129

Letarte, B., Demers, S., Battinelli, P., \& Kunkel, W. E. 2002, AJ, 123, 832

Maylan, G., Sarajedini, A., Jalonka, P., et al. 2001, AJ, 122, 830

Racine, R. 1991, AJ, 101, 865

Reitzel, D. B., Guhatakhurta, P., \& Rich, R. M. 2004, AJ, 127, 2133

Rich, M. R., Mighell, K. J., Freedman, W. D., \& Neill, J. D. 1996, AJ, 111,768

Rich, M. R., Reitzel, D. B., Guhathakurta, P., et al. 2004, AJ, 127, 2139

Richer, H. B. 1981, ApJ, 243, 744

Richer, H. B., Olander, N., \& Westerlund, B. E. 1979, ApJ, 230, 724

Richer, H. B., Westerlund, B. E., \& Olander, N. 1978, ApJ, 219, 452

Walterbos, R. A. M., \& Kennicutt, R. C. 1988, A\&A, 198, 61 